\title{
Buccinator Myomucosal Flap for Treatment of Osteoradionecrosis of the Mandible
}

\author{
Seung Hoon Woo ${ }^{1,2}$ \\ ${ }^{1}$ Department of Otorhinolaryngology, ${ }^{2}$ Institute of Health Sciences, Gyeongsang National University School of Medicine, Jinju, Korea
}

\begin{abstract}
The use of a myomucosal flap from the buccinator muscle is a valuable reconstruction method for intraoral defects. In this paper, we report the clinical advantages of using a buccinator myomucosal flap for the treatment of partial mandibular defects caused by osteoradionecrosis. We implemented a buccinator myomucosal flap for the reconstruction of a partial mandibular defect in a 55-year-old man with tonsil cancer and partial mandibular defects caused by osteoradionecrosis. The total operating time was 90 minutes. Twelve months after the reconstruction, the patient remains free of disease. A buccinator myomucosal flap can be used for the reconstruction of partial mandibular defects caused by osteoradionecrosis. It is a reliable method for reconstructing small mandibular defects.
\end{abstract}

Keywords. Osteoradionecrosis; Myocutaneous Flap; Reconstructive Surgical Procedures; Mandibular Reconstruction

\section{INTRODUCTION}

Mandibular osteoradionecrosis (ORN) is a potentially debilitating complication from ionizing radiotherapy, whose incidence is reported to range between $5 \%$ and $15 \%$. ORN is most commonly defined as an exposed, irradiated, nonhealing defect of the mandible with symptoms lasting at least 2 to 3 months, without any evidence of tumor recurrence. Although this complication often arises in the first 6 to 12 months after radiotherapy, reports of late incidence suggest a lifelong risk. The risk factors for ORN include a high radiation dose (>60 Gy), previous dental extraction, radiation to the posterior mandible, infection, a large tumor size, malnutrition, poor oral hygiene, and alcohol and tobacco abuse. Although the condition is most often associated with dental extractions or mucosal trauma, spontaneous cases of ORN have been recorded.

The treatment for ORN was first designed in the 1950s and comprises a multimodal approach utilizing conservative measures such as antibiotic therapy, debridement, and irrigation in

\footnotetext{
- Received November 19, 2014

Revised December 26, 2014

Accepted January 14, 2015

- Corresponding author: Seung Hoon Woo

Department of Otorhinolaryngology, Gyeongsang National University

Hospital, Gyeongsang National University School of Medicine,

79 Gangnam-ro, Jinju 52727, Korea

Tel: +82-55-750-8173, Fax: +82-55-759-0613

E-mail: lesaby@hanmail.net
}

the early stages of the disease, while reserving surgical resection and reconstruction for more advanced or refractory cases. Recently, interest in the management of ORN has been rekindled with the introduction of newer reconstructive techniques [1-5]. The purpose of this presentation is to describe our recent experience with a buccinator myomucosal flap implemented for the reconstruction of a partial mandibular defect caused by ORN.

\section{CASE REPORT}

A 55-year-old male patient visited at Gyeongsang National University Hospital with a complaint of tonsil cancer. The patient was preparing to undergo concurrent chemoradiotherapy for human papillomavirus-positive cancer. He was experiencing problems with his left molar tooth, and the latter was therefore extracted before the patient underwent the next radiation therapy session (after sufficient time had elapsed). The tonsil cancer was treated with radiation therapy of 7,200 rad. However, a defect appeared in the region where the left molar tooth had been extracted (Fig. 1A). The defect was diagnosed as a case of ORN caused by the radiation therapy, and antibiotic administration and symptomatic therapy were followed for 3 months. However, the defect size gradually increased and the necrosis region grew wider, calling for a different type of treatment.

On the basis of our experience with tongue reconstruction using a buccinator flap, we planned the reconstruction of the de-

Copyright @ 2016 by Korean Society of Otorhinolaryngology-Head and Neck Surgery.

This is an open-access article distributed under the terms of the Creative Commons Attribution Non-Commercial License (http://creativecommons.org/licenses/by-nc/4.0)

which permits unrestricted non-commercial use, distribution, and reproduction in any medium, provided the original work is properly cited. 

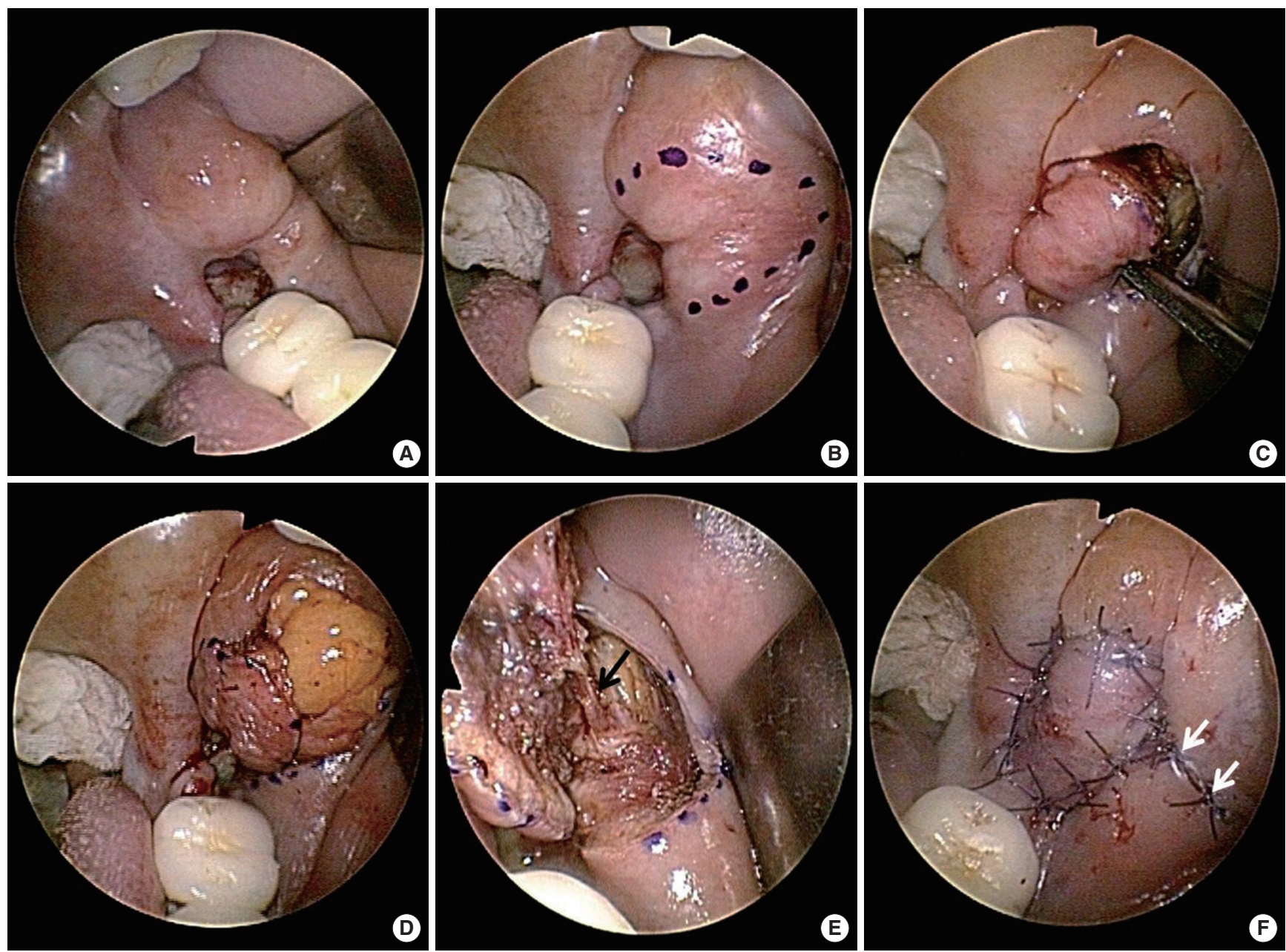

Fig. 1. (A) Osteoradionecrosis at the left molar extraction site. (B) Drawing the incision for the harvesting of the buccinator flap. (C) Buccinator flap harvesting. (D) Harvesting of the buccal fat with the buccinator flap. (E) Foundation of the neurovascular pedicle (arrow). (F) Defect area filled with buccal fat and buccinator flap. The donor site was closed with primary suture well-healed (arrows).

fect site using a buccinator artery-based buccal flap. After sufficiently sterilizing the defective area, the necrosis region was ground with a drill and was removed in order to view the fresh tissue. Afterwards, an incision slightly larger than the defect site was performed in the buccal mucosa (Fig. 1B). After incising the buccal mucosa and the muscle along the upper and anterior borders, the flap was elevated in an anterior to posterior direction (Fig. 1C). When harvesting the buccinator flap, the buccal fat layer was also harvested so that the defect site in the mandible could be obliterated (Fig. 1D). The neurovascular pedicle (including the buccal artery, vein, and nerve) was identified (Fig. 1E). The dissection was continued inferiorly and laterally, and lower and posterior incisions were made so that the flap could be elevated. The flap was then rotated to fill the defect site with buccal fat, and the harvested buccal mucosa was sutured to the mucosa of the defect site (Fig. 1F). Subsequently, the donor site was sutured with primary suture. The total operating time was 90 minutes. One week after the operation, good settlement of
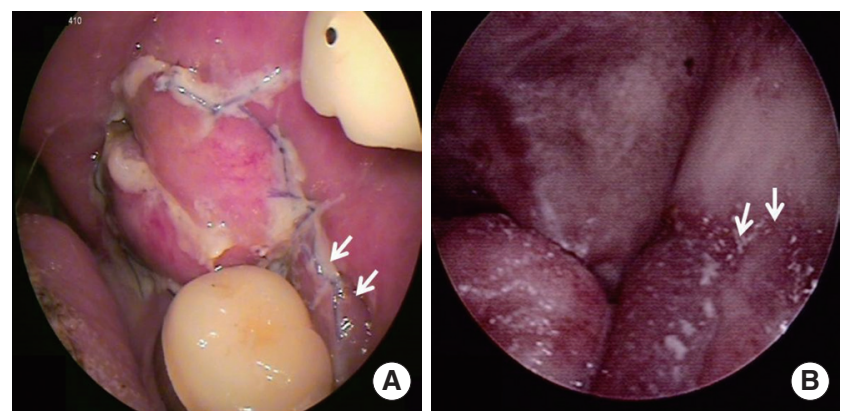

Fig. 2. (A) One week after surgery, the linear scar was excised at the donor site (arrows). (B) Twelve months after surgery, the donor site and recipient site were well-healed (arrows).

the flap was observed (Fig. 2A). One year later, it was found that the donor site and the recipient site were stably healed (Fig. 2B). The patient was healed without particular discomfort, and the ORN did not progress any further. 


\section{DISCUSSION}

Clinicians will agree that injuries of the mandible caused by radiation remain a challenging problem, and can be more difficult to manage than the original cancer. The disastrous effects of ORN of the mandible, including orocutaneous fistulae, pathologic fractures, associated soft-tissue necrosis, and trismus, are clearly much easier to prevent than to treat $[5,6]$.

Early theories on ORN held that three factors-radiation, trauma, and infection - contributed to the pathophysiology of this disease process. Marx subsequently promoted his 3-H paradigm, whereby radiation induced tissue injuries by causing vessel thrombosis (hypovascularity), leading to hypoxia and the resultant cell death of the skin/mucosa and underlying bony element (hypocellularity). This theory supported the potential therapeutic role of hyperbaric oxygen therapy (HBO) therapy, which Marx incorporated into a standardized treatment protocol (Wilford Hall HBO ORN protocol) [7]. ORN is, therefore, a problem of impaired and inadequate tissue turnover and wound healing [7].

No widely-accepted, evidence-based guidelines for the use of HBO therapy in the prevention and management of ORN have been established. Protocols utilizing HBO therapy, which were once considered the standard of care, are no longer recommended for the prevention or treatment of ORN $[8,9]$. Newer theories describing the pathogenesis of ORN have stimulated the evolution of conservative management strategies, including the administration of drugs such as pentoxifylline and tocopherol $[9,10]$. Bony replacement with flap reconstruction is appropriate for patients with ORN who fail to respond to conservative treatment [9].

Nowadays, the primary reconstruction of mandibular defects which have failed to be resolved by conservative treatment is desirable, and even mandatory. With the advent of microsurgical techniques, free vascularized grafts have become the preferred method for reconstructing mandibular defects. In fact, a vascularized free fibular flap represents an optimal reconstruction method after a mandibular resection [11-14]. However, the free flap is difficult and slow to perform, and causes a problem of morbidity of the donor site. Therefore, if the mandibular defect is small, a regional pedicle flap from the same oral cavity may be used as an easier way to reduce the duration of the operation and to remove the problem of morbidity of the donor site. Thus, if the mandibular defect is not large, an adjacent buccinator myomucosal flap supplied by the buccal artery may be more useful. We recently reported that the tongue may be reconstructed using a buccinator myomucosal flap, and the present case reports an advanced method of reconstruction [15].

When a buccinator myomucosal flap is used, only a linear scar remains at the donor site, and the bony defect of the mandible is filled with harvested buccal fat, which can prevent the progress of the bony defect. Moreover, since the same oral mucosa is used as tissue replacement, contracture can be prevented.

Research on the reconstruction of mandibular defects caused by ORN using a buccinator myomucosal flap supplied by the buccal artery has just started. Therefore, there is no confirmation that all ORNs can be treated with a buccinator myomucosal flap. However, the present report is significant in that an opportunity was created to consider an alternative treatment method to conservative management and free flap surgery.

Our results reveal that a buccinator myomucosal flap can be performed for reconstruction of a partial mandibular defect caused by ORN, and that the buccinator myomucosal flap is a reliable method to reconstruct small mandibular defects.

\section{CONFLICT OF INTEREST}

No potential conflict of interest relevant to this article was reported.

\section{ACKNOWLEDGMENTS}

This research was supported by the Basic Science Research Program through the National Research Foundation of Korea (NRF) funded by the Ministry of Science, ICT, and Future Planning (2013R1A1A1012542). This research was supported by the Leading Foreign Research Institute Recruitment Program through the National Research Foundation of Korea (NRF) funded by the Ministry of Education, Science and Technology (MEST) (2012K1A4A3053142).

\section{REFERENCES}

1. Hidalgo DA. Fibula free flap: a new method of mandible reconstruction. Plast Reconstr Surg. 1989 Jul;84(1):71-9.

2. Hidalgo DA. Fibula free flap mandibular reconstruction. Clin Plast Surg. 1994 Jan;21(1):25-35.

3. Marx RE, Johnson RP, Kline SN. Prevention of osteoradionecrosis: a randomized prospective clinical trial of hyperbaric oxygen versus penicillin. J Am Dent Assoc. 1985 Jul;111(1):49-54.

4. Sanger JR, Matloub HS, Yousif NJ, Larson DL. Management of osteoradionecrosis of the mandible. Clin Plast Surg. 1993 Jul;20(3): 517-30.

5. Shaha AR, Cordeiro PG, Hidalgo DA, Spiro RH, Strong EW, Zlotolow I, et al. Resection and immediate microvascular reconstruction in the management of osteoradionecrosis of the mandible. Head Neck. 1997 Aug;19(5):406-11.

6. Ang E, Black C, Irish J, Brown DH, Gullane P, O'Sullivan B, et al. Reconstructive options in the treatment of osteoradionecrosis of the craniomaxillofacial skeleton. Br J Plast Surg. 2003 Mar;56(2):92-9.

7. Marx RE. Osteoradionecrosis: a new concept of its pathophysiology. J Oral Maxillofac Surg. 1983 May;41(5):283-8.

8. Annane D, Depondt J, Aubert P, Villart M, Gehanno P, Gajdos P, et al. Hyperbaric oxygen therapy for radionecrosis of the jaw: a randomized, placebo-controlled, double-blind trial from the ORN96 study group. J Clin Oncol. 2004 Dec 15;22(24):4893-900.

9. Lubek JE, Hancock MK, Strome SE. What is the value of hyperbaric 
oxygen therapy in management of osteoradionecrosis of the head and neck? Laryngoscope. 2013 Mar;123(3):555-6.

10. Delanian S, Chatel C, Porcher R, Depondt J, Lefaix JL. Complete restoration of refractory mandibular osteoradionecrosis by prolonged treatment with a pentoxifylline-tocopherol-clodronate combination (PENTOCLO): a phase II trial. Int J Radiat Oncol Biol Phys. 2011 Jul 1;80(3):832-9.

11. Serafin D, Villarreal-Rios A, Georgiade NG. A rib-containing free flap to reconstruct mandibular defects. Br J Plast Surg. 1977 Oct;30(4): 263-6.

12. Swartz WM, Banis JC, Newton ED, Ramasastry SS, Jones NF, Acland
R.The osteocutaneous scapular flap for mandibular and maxillary reconstruction. Plast Reconstr Surg. 1986 Apr;77(4):530-45.

13. Taylor GI. Reconstruction of the mandible with free composite iliac bone grafts. Ann Plast Surg. 1982 Nov;9(5):361-76.

14. Kim JP, Park HW, Park JJ,Woo SH. Reconstruction after resection of a lower lip squamous cell carcinoma with a submental island flap. Ear Nose Throat J. 2015 Dec;94(12):E19-21.

15. Woo SH, Jeong HS, Kim JP, Park JJ, Ryu J, Baek CH. Buccinator myomucosal flap for reconstruction of glossectomy defects. Otolaryngol Head Neck Surg. 2013 Aug;149(2):226-31. 